\title{
Um sistema baseado em regras fuzzy para caracterizar o perfil de um investidor
}

\author{
A fuzzy rule-based system to characterize the profile of an investor
}

\author{
Tais Mara dos Santos ${ }^{\prime}$ \\ Michele Cristina Valentino" \\ Douglas Azevedo III \\ Danilo Peixoto Bellucci Iv
}

\section{Resumo}

Neste trabalho é proposto um sistema de classificação do perfil de um investidor por meio de base de regras fuzzy. Para tal, é utilizado o ambiente GUIDE do Matlab, para construir uma interface interativa, no qual são fornecidas, pelo potencial investidor, respostas para cinco questões (baseadas no modelo da ANBIMA), que versam sobre suas finanças. A partir destas respostas, o sistema apresenta como saída uma das características: investidor conservador, investidor moderado ou investidor arrojado, indicando também uma sugestão do tipo de investimento que melhor se enquadra a seu perfil. Os resultados obtidos são comparados com os resultados obtidos pelo API do Banco Paulista.

Palavras-chave: Classificação do perfil de investidor; Regras fuzzy; Matlab

\section{Abstract}

In this work is proposed a fuzzy rule-based system in order to classify the profile of an investor. For this purpose, it is used the plataform GUIDE from MATLAB to build an interactive graphic interface, where the investor answers five questions (which are based on the ANBIMA model) which deal with his/her finances. Once the system has these answers, it presents as output one of the following classifications: conservative investor, moderate investor or bold investor. The system also indicates a suggestion of potentials types of investments that suits better based on the output provided by the system. The results are compared to the ones obtained by the AIP from the Banco Paulista.

Keywords: Classification of the profile of an investor; Fuzzy rules; Matlab 


\section{Introdução}

Em 1965 Zadeh publicou os primeiros resultados da teoria fuzzy, os quais são capazes de produzir cálculos com informações imprecisas que encontramos nas várias situações do dia-a-dia. Desde então, esta teoria tem sido utilizada para modelar problemas em vários ramos da ciência, como por exemplo para estimar valores de ações da Bolsa de Valores de São Paulo e obter assim as melhores opções de investimento Cichini (2009), para analisar a ocorrência dos casos de Malária no estado do Amazonas de 2009 à 2015 Santos e Prata (2016), para controlar o desempenho de uma turbina eólica de velocidade variável com a finalidade de contirbuir para a melhoria do desempenho dinâmico da turbina no tocante a redução de oscilações, máximo sobresinal e tempo de acomodação Sena (2015), para classificar uma pessoa com relação a sua aptidão ao empreendedoristo Bellucci e Brandão (2010) e etc.

O problema de classificar um investidor possui muitas subjetividades. Atualmente, antes de qualquer banco indicar algum investimento a um futuro investidor, é exigido pela Associação Brasileira das Entidades dos Mercados Financeiro e de Capitais (ANBIMA) que o cliente responda um questionário, denominado Análise do Perfil do Investidor (API), com algumas perguntas que o classifica como conservador, moderado ou arrojado e então o auxilia a descobrir quais são os investimentos mais adequados ao seu perfil. O guia de melhores práticas da ANBIMA, reconhece que para uma melhor definição do perfil do investidor, é essencial que sejam abordados os seguintes aspectos: tolerância ao risco, experiência em investimentos, objetivos e horizonte de tempo.

Com a finalidade de dar tratamento matemático aos termos subjetivos do problema de classificar um investidor, neste trabalho será construído um modelo teórico baseado em regras fuzzy, Barros e Bassanezi (2006), que auxiliará um investidor na escolha dos melhores investimentos para o seu perfil. Para isso, será utilizado o Fuzzy Logic Toolbox do MATLAB (MathWorks Inc. (2016)) e a elaboração das funções de pertinência bem como da base de regras serão produzidas com o auxílio de um especialista na área de finanças.

\section{Teoria Fuzzy e Sistema de Classificação Fuzzy}

Nesta seção serão apresentadas algumas definições, as quais serão úteis para compreender a principal modelagem deste trabalho. Mais resultados podem ser encontrados em (Barros e Bassanezi, 2006).

Definição 1. Seja $X$ um conjunto clássico não vazio. Um subconjunto fuzzy $A$ de $X$ é caracterizado por uma função $\varphi_{\mathrm{A}}: X \rightarrow[0,1]$, chamada função de pertinência do conjunto fuzzy A. O valor $\varphi_{A}(x) \in[0,1]$ indica o nível que o elemento $x$ de $X$ pertence ao conjunto fuzzy $A$, ou seja , quando $\varphi_{\mathrm{A}}(x)=0$ tem-se que o elemento $x$ não pertence ao conjunto e quando $\varphi_{\mathrm{A}}(x)=1$ tem-se que o elemento $x$ pertence totalmente no conjunto fuzzy .

Nas próximas definições são apresentadas as opera- ções entre os subconjuntos fuzzy.

Definição 2. A união de $A$ e $B$ é o subconjunto fuzzy de $U$ cuja função de pertinência é dada por:

$$
\varphi(A \cup B)(x)=\max \{\varphi A(x), \varphi B(x)\}, x \in U .
$$

Definição 3. A intersecção entre A e B é o subconjunto fuzzy de U cuja função de pertinência é dada por:

$$
\varphi(A \cap B)(x)=\min \{\varphi A(x), \varphi B(x)\}, x \in U .
$$

Definição 4. O complementar de A é o subconjunto fuzzy A' de U cuja função de pertinência é dada por:

$$
\varphi A^{\prime}(x)=1-\varphi A(x), x \in U \text {. }
$$

Na lógica fuzzy, os conectivos lógicos e, ou e então são modelados pelos operadores $\Delta$ (t-norma), $\nabla$ (t-conorma), $\Rightarrow$, respectivamente, em que neste trabalho serão considerados $\Delta(x, y)=\min \{x, y\}=x \wedge y$ e $\nabla(x, y)=\max \{x, y\}=\mathrm{x} \vee \mathrm{y}$.

Definição 5. Uma relação fuzzy $\Re$, sobre $U_{1} \times U_{2} \times \cdots \times U_{n}$ é qualquer subconjunto fuzzy do produto cartesiano $U_{1} \times U_{2} \times \cdots \times U_{n}$. Assim, $\mathfrak{R}$ é definida por uma função de pertinência $\Re \varphi: U_{1} \times U_{2} \times \cdots \times U_{n} \rightarrow[0,1]$, a qual indica o grau com que os elementos $x_{i}$, que compõem a n-upla $\left(x_{1}, x_{2}, \ldots, x_{i}\right)$ estão relacionados segundo a relação .

Definição 6. O produto cartesiano fuzzy dos subconjuntos fuzzy $A_{1}, A_{2} \ldots, A_{n}$ de $U_{1}, U_{2}, \ldots, U_{n}$, respectivamente, é a relação fuzzy de $A_{1} \times A_{2} \times \ldots \times A_{n}$, cuja função de pertinência é dada por:

$$
\begin{aligned}
& \varphi_{A_{1} \times A_{2} \times \cdots \times A_{n}}\left(x_{1}, x_{2}, \cdots, x_{n}\right)= \\
& =\varphi_{\mathrm{A}_{1}}\left(x_{1}\right) \wedge \varphi_{\mathrm{A}_{2}}\left(x_{2}\right) \wedge \cdots \wedge \varphi_{\mathrm{A}_{\mathrm{n}}}\left(x_{n}\right),
\end{aligned}
$$

\section{Onde $\wedge$ é o operador $\Delta$.}

A combinação de duas ou mais relações fuzzy pode ser feita através de operadores que permitem a composição das mesmas. A seguir será definida a composição utilizada neste trabalho.

Definição 7. Considere que $\mathfrak{R}$ e $S$ são as relações fuzzy $U \times V$ e $V \times W$, respectivamente. A composição $R \circ S$ é uma relação fuzzy em $U \times W$ cuja função de pertinência é dada por:

$$
\varphi_{\mathcal{R} \circ \mathcal{S}}(u, w)=\sup _{v \in V}\left\{\min \left\{\varphi_{\mathcal{R}}(u, v), \varphi_{\mathcal{S}}(u, w)\right\}\right\} .
$$

Um sistema baseado em regras fuzzy utiliza a lógica fuzzy para obter saída para cada entrada fuzzy. Um caso particular desse sistema é chamado de Classificador Fuzzy. No método de classificação tradicional tem-se que cada dado é membro de uma classe ou não, já na classificação fuzzy temos que um dado pode pertencer a várias classes ao mesmo tempo e com diferentes graus de pertinência. O classificador fuzzy baseado em regras consiste de regras fuzzy, as quais descrevem uma das classes de saída. Ainda é composto pelos módulos: fuzzificação, base de regras, inferência fuzzy e defuzzificação. 
Fuzzificação: A fuzzificação modela matematicamente as informações das variáveis de entrada através de conjuntos fuzzy. A realização dessa etapa deve ser feita com a ajuda de um especialista, na falta do especialista pode-se recorrer a treinamentos supervisionados, a partir do conjunto de dados, como as redes neurais por exemplo.

Base de Regras: A base de regras cumpre o papel de "traduzir" matematicamente as informações que formam a base de conhecimento do sistema fuzzy. Pode ser dada da seguinte forma:

\section{Se x é Aj Então $u$ é Bj,}

e são construídas de acordo com as combinações das variáveis de entrada e suas respectivas funções de pertinência. A base de conhecimento é composta por todas as combinações possíveis, por exemplo, para duas entradas, cujas funções de pertinência são dadas por valores linguísticos baixo, médio e alto, então a base de conhecimento será composta por $3^{2}$ regras.

Inferência Fuzzy: Nesse módulo são escolhidos quais são os conectivos lógicos mais adequados na obtenção da relação fuzzy que modela a base de regra, que é o responsável por fornecer o comportamento da variável de saída fuzzy, através de um conjunto finito dessas regras mediante processo de inferência fuzzy. Na aplicação que será apresentada neste trabalho será utilizado o método de inferência de Mamdani, que pode ser exemplificado pelo seguinte procedimento:

- Em cada regra $R_{j}$ da base de regras fuzzy, a condicional "se $x$ é $A_{i}$ então $u$ é $B_{j}$ " é modelada por $\wedge$ (mínimo);

- Adota-se a t-norma $\wedge$ (mínimo) para o conectivo lógico "e";

- Para o conectivo lógico "ou" adota-se a t-conorma $v$ (máximo) que conecta as regras fuzzy da base de regras.

Com base em (Barros e Bassanezi, 2006), a relação fuzzy $M$ é o subconjunto fuzzy de $X \times \mathrm{U}$, cuja função de

$\varphi_{\mathcal{M}}(x, u)=\max _{1 \leq j \leq r}\left\{\varphi_{R_{j}}(x, u)\right\}=\max _{1 \leq j \leq r}\left\{\varphi_{A_{j}}(x) \wedge \varphi_{B_{j}}(u)\right\}$

pertinência é dada por

em que $r$ é o número de regras que compõem a base de regras, e $A_{j}$ e $B_{j}$ são os subconjuntos fuzzy da regra $j$. Cada um dos valores $\varphi_{A \mathrm{~A}}(\mathrm{x})$ e $\varphi_{\mathrm{B}}(\mathrm{u})$ são interpretados como o grau com que $x$ e $u$ pertencem aos subconjuntos fuzzy $A_{i}$ e $B_{i}$, respectivamente. Assim, essa relação pode ser resumida como sendo a união dos produtos cartesianos fuzzy entre os antecedentes e os consequentes de cada regra.

Defuzzificação: O processo de defuzzificação, permite transformar uma variável de saída fuzzy em um valor numérico. São muitos os métodos de defuzzificação que podem ser utilizados, os mais comuns são, centro de gravidade, centro dos máximos e média dos máximos. Para a aplicação apresentada neste trabalho será utilizado o módulo de defuzzificação centro de gravidade, que é
$G(B)=\frac{\sum_{i=0}^{n} u_{i} \varphi_{B}\left(u_{i}\right)}{\sum_{i=0}^{n} \varphi_{B}\left(u_{i}\right)}$

calculado da seguinte forma:

$G(B)=\frac{\int_{\mathbb{R}} u \varphi_{B}(u) d u}{\int_{\mathbb{R}} \varphi_{B}(u) d u}$

quando o domínio for discreto, e se o domínio for contínuo.

Na próxima seção, será apresentada uma aplicação utilizando sistemas de classificação fuzzy baseado em regras, a qual poderá auxiliar o investidor no processo de tomada de decisão de um investimento bancário.

\section{Classificação de um investidor via} sistema baseado em regras fuzzy

Existem vários fatores que influenciam o investidor antes da tomada de decisão de um investimento bancário. A escolha de um investimento é muito importante, pois tanto no curto ou longo prazo, essa escolha pode interferir nos seus planos caso a decisão tomada não seja a certa. Para ajudar na escolha do investimento bancário mais adequado a cada tipo de perfil, os bancos propõem ao investidor um formulário, que são conhecidos como análise do perfil do investidor (API), eles são respondidos no início de todo o processo, com o objetivo de classificar o investidor e depois oferecer os tipos de investimentos adequados.

Segundo Portal do Investidor (2016) os perfis dos investidores podem ser classificados da seguinte maneira:

- Investidor Conservador: privilegia a segurança e faz todo o possível para diminuir o risco de perdas, para isso ele até aceita uma rentabilidade menor.

- Investidor Moderado: investe mais no longo prazo, buscando retorno superior à renda fixa, correndo mais riscos que o conservador.

- Investidor Arrojado: privilegia a rentabilidade e é capaz de correr grandes riscos para que seu investimento renda o máximo possível.

Levando em consideração os aspectos já citados anteriormente que são exigidos pela ANBIMA e com base nos estudos da API do banco Paulista Banco Paulista (2016) foram escolhidas as seguintes variáveis de entrada (Pergunta feita ao possível investidor):

- Carência do investimento (Por quanto tempo você pretende deixar o seu dinheiro investido sem que ele possa ser resgatado? (meses));

- Conhecimento no mercado financeiro (Dê uma nota a você mesmo, de 0 a 100, a qual representa seu conhecimento no mercado financeiro.);

- $\quad$ Aversão ao risco (Dê uma nota a você mesmo, de 0 a 100, a qual indica o quanto você está apto a arriscar.); 
- $\quad$ Realizar mais aplicações (Dê uma nota a você mesmo, de 0 a 100, a qual indica a possibilidade de você realizar mais aplicações, mesmo se seus rendimentos não renderem o esperado no curto prazo.);

- Prazo do retorno esperado (Em quanto tempo você espera que sua aplicação gere resultado? (meses))
Com a ajuda do especialista, Danilo Peixoto Bellucci ${ }^{1}$, foram elaborados os parâmetros das funções de pertinência das variáveis de entrada do sistema, as funções do tipo trapezoidal foram selecionadas para representar as funções de pertinência, pois de acordo com o especialista eram as mais adequadas para modelar as variáveis do problema, visto que há um período de estabilidade.

Figura 1 - (a) Função de pertinência da variável "carência do investimento", (b) Função de pertinência da variável "conhecimento no mercado financeiro".
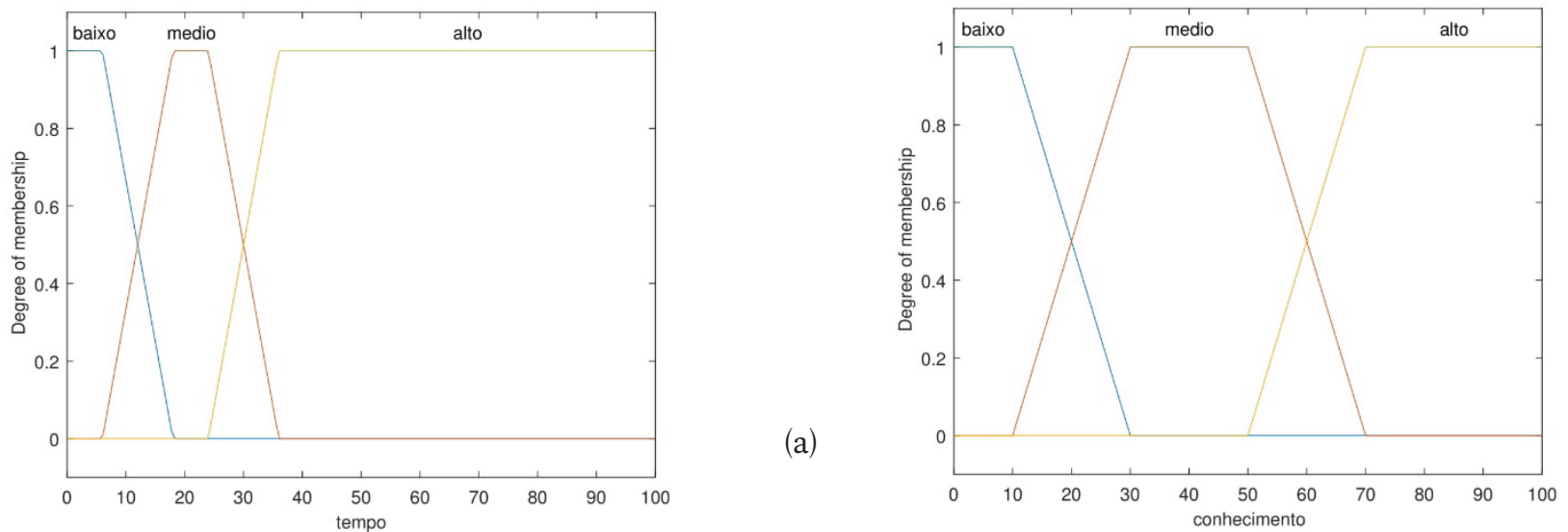

(b)

Figura 2 - (a) Função de pertinência da variável: "aversão ao risco", (b) Função de pertinência da variável: "realizar mais aplicações", (c) Função de pertinência da variável: "prazo do retorno esperado".
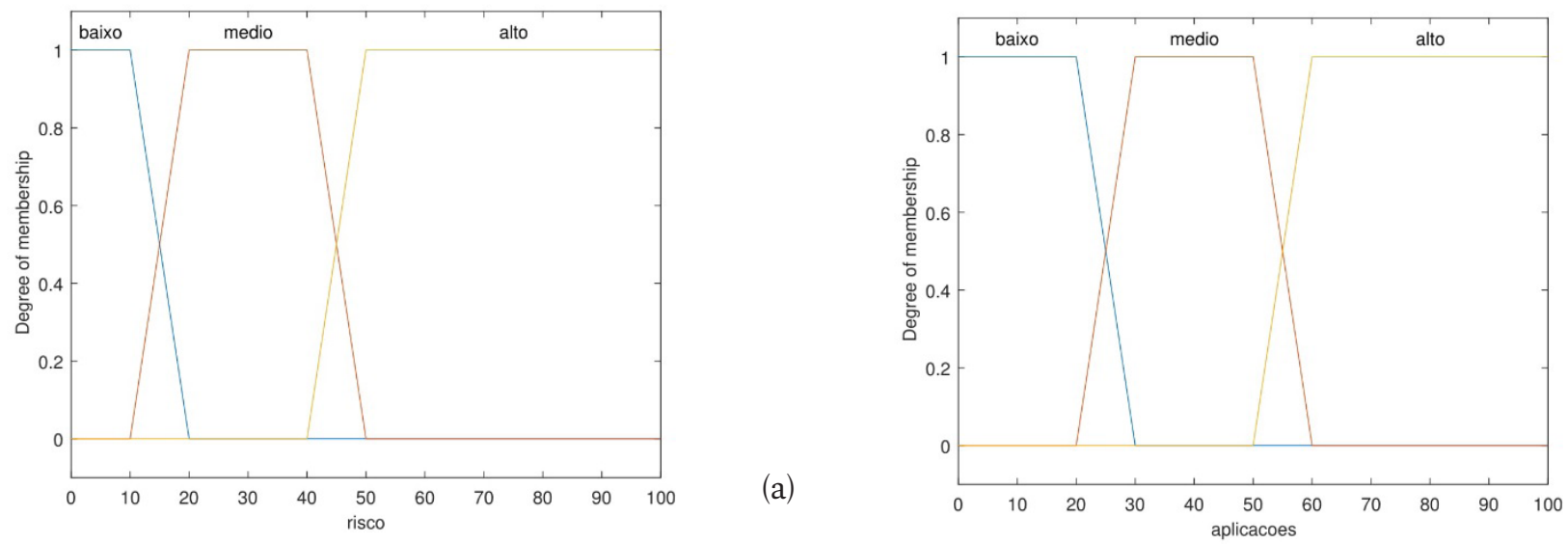

(b)

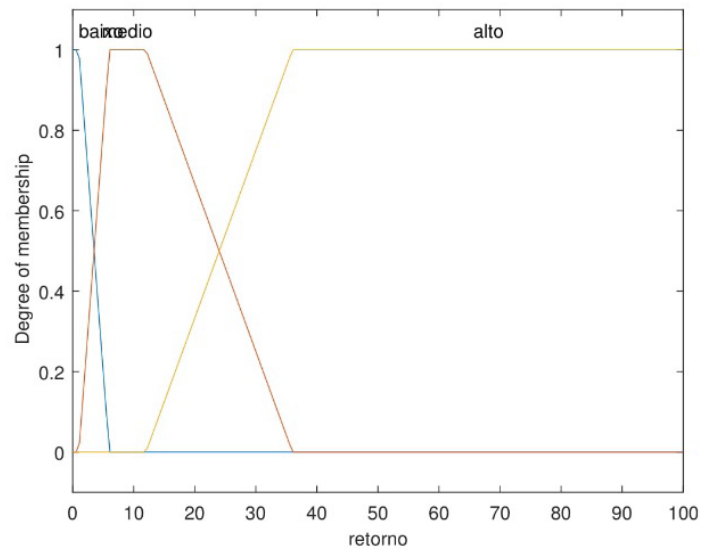

(c) 
Tabela 1 - Base de regras linguísticas

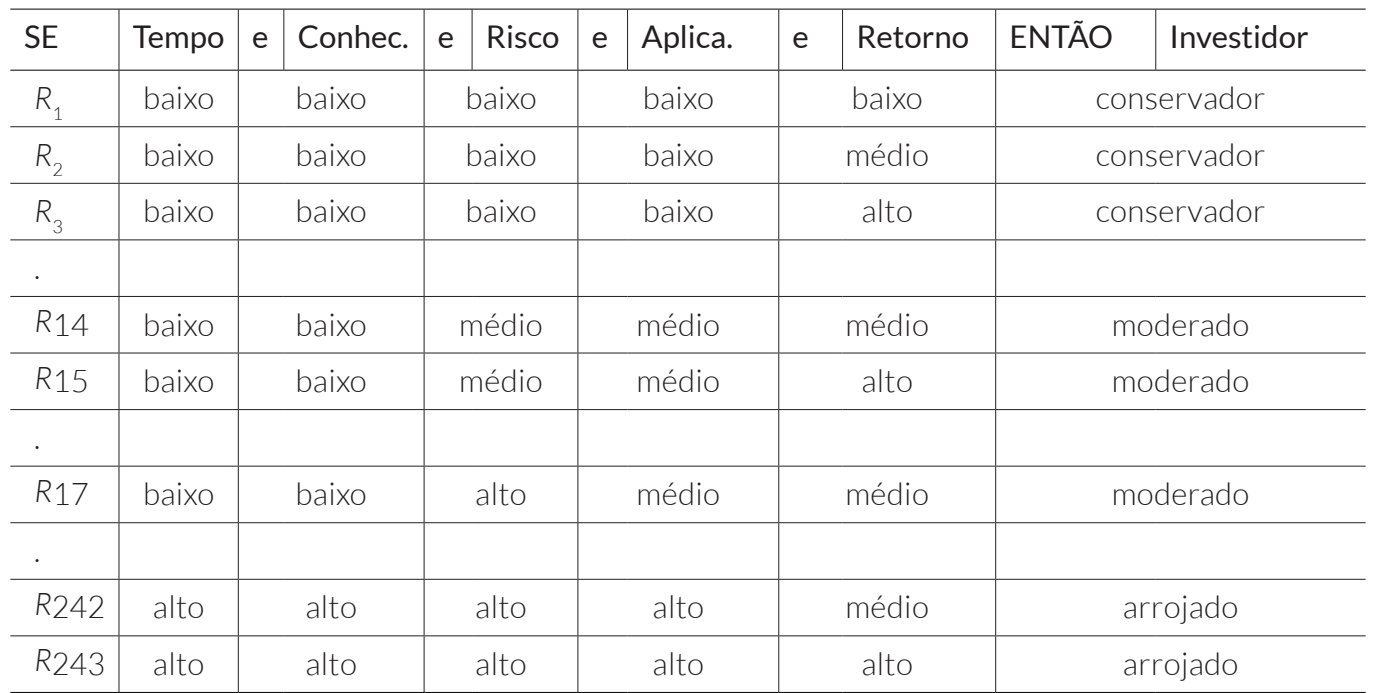

As Figuras 1 e 2 mostram as funções de pertinência das variáveis de entrada.

Figura 3 - Função de pertinência da variável de saída.

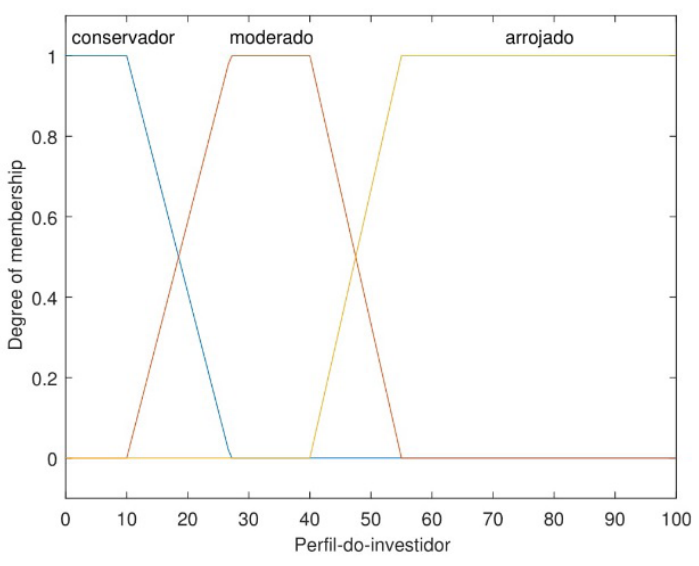

A Figura 3 representa a função de pertinência da variável de saída "classificação do investidor", a qual é atrubuída os seguintes valores linguísticos "conservador", "moderado" e "arrojado".

O modelo foi desenvolvido no FIS editor do Fuzzy Logic Toolbox, que é uma ferramenta do software MATLAB. Ainda, foi utilizado o método de inferência de Mamdani para obter a relação fuzzy que modela a base de regras e o método de defuzzificação do centro de gravidade para obter um valor numérico que representará a saída do sistema.

Na Tabela 1 são apresentados alguns exemplos das 243 regras linguísticas utilizadas na aplicação.

\section{Resultados}

Na Figura 4 encontramos a interface gráfica do API construída no GUIDE do MATLAB. Nesta janela, o interessado responde as perguntas do API que são as entradas do sistema, e então o modelo fornece a variável de saída defuzzificada que é o perfil do investidor. A interface ainda fornece alguns investimentos sugeridos de acordo com o perfil do investidor.

O API do Banco Paulista, o qual foi utilizado como referência para comparações com relação ao sistema baseado em regras fuzzy aqui apresentado, baseia-se num questionário com 8 questões de múltipla escolha, baseadas nas exigências da ANBIMA. Este questionário tem por objetivo caracterizar o perfil de um investidor com base nas respostas dadas as tais questões.

A seguir, são apresentadas algumas comparações entre o API do Banco Paulista e o sistema baseado em regras fuzzy desenvolvido neste trabalho. Utilizaremos a notação 2-a para indicar que na questão $2 \mathrm{o}$ item a do API do Banco Paulista (Banco Paulista, 2016), foi as resposta fornecida.

$1^{\text {a }}$ Comparação As respostas do Investidor 1 para o API do Banco Paulista são:

$$
\text { 1-d, 2- a, 3- a, 4- a, 5- a, 6- b, 7- a e 8- a, }
$$

com essas respostas foi obtida uma pontuação igual a 6, assim o mesmo investidor foi classificado como um investidor conservador, segundo o API do Banco Paulista. Em seguida, foi pedido para o mesmo responder o API desenvolvido neste trabalho, ou seja, as questões indicadas na Figura $4 \mathrm{e}$, neste caso, as respostas fornecidas foram: $48,1,10,10,24$. A classificação do investidor pelo novo sistema foi de 11.2481, por isso, de acordo com a função de pertinência da variável de saída (veja Figura 3) tal investidor foi classificado como um investidor conservador e moderado, no entanto nota-se que há uma pertinência consideravelmente maior com relação ao perfil conservador.

$2^{\mathbf{a}}$ Comparação As respostas do Investidor 2 para o API do Banco Paulista são:

1- c, 2- b, 3- a, 4- a, 5- b, 6- b, 7- a e 8- b, 
Figura 4 - Interface gráfica.

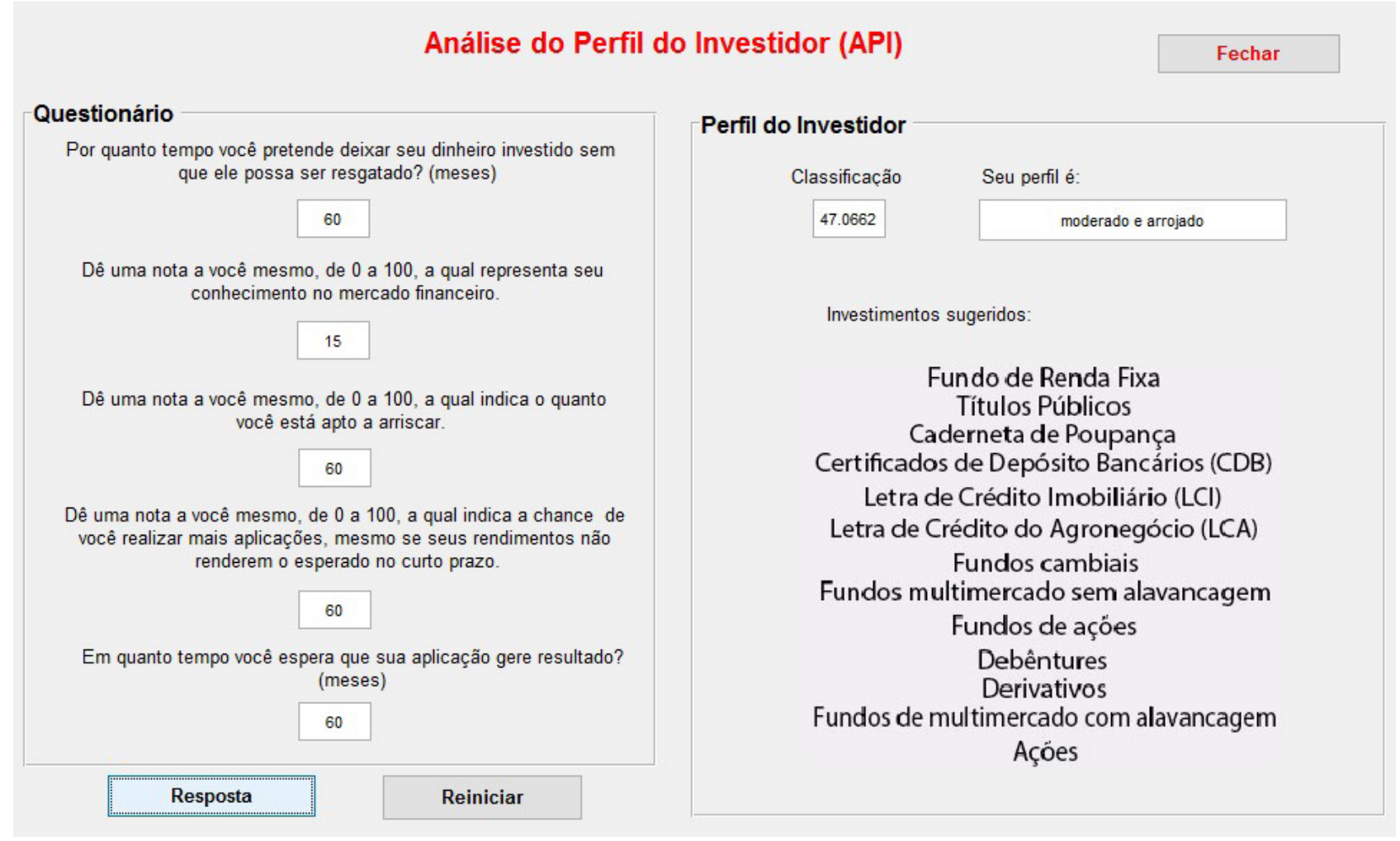

com essas respostas foi obtida uma pontuação igual a 11, logo foi classificado como um investidor moderado. Em seguida, foi pedido para o mesmo responder o API desenvolvido neste trabalho, ou seja, as questões indicadas na Figura $4 \mathrm{e}$, neste caso, as respostas fornecidas foram: 24, $20,40,60,24$. A classificação do investidor foi de 32.731 e por esse motivo este investidor foi classificado como um investidor moderado (veja Figura 3). Portanto, investimentos do perfil moderado serão sugeridos a esse investidor.

$3^{\mathbf{a}}$ Comparação As respostas do Investidor 3 para o API do Banco Paulista são:

$$
\text { 1- c, 2- b, 3- a, 4- a, 5- a, 6- b, 7- a e 8- b, }
$$

com essas respostas foi obtida uma pontuação igual a 9, logo foi classificado como um investidor conservador. Em seguida, foi pedido para o mesmo responder o API desenvolvido neste trabalho, ou seja, as questões indicadas na Figura $4 \mathrm{e}$, neste caso, as respostas fornecidas foram: 24 , $20,30,06,12$. A classificação do investidor foi de 25.4121 e por isso, de acordo com a função de pertinência da variável de saída (veja Figura 3) o mesmo foi classificado como um investidor conservador e moderado, com grau de pertinência maior para o perfil moderado.

Após análise das respostas fornecidas a ambos APIs, constata-se que a diferença entre as classificações apresentadas pelos APIs ocorreu devido a resposta 4-a, fornecida ao API do Banco Paulista, a qual afirma que o investidor não possui formação acadêmica ou conhecimento no mercado financeiro, uma vez que, no API desenvolvido neste trabalho o mesmo investidor indicou nota 20 com relações ao conhecimento no mercado financeiro, ou seja, foi indicado que o mesmo tinha algum conhecimento no tema. Se o mesmo tivesse indicado nota menor, por exemplo 14, ele seria classificado como conservador e moderado, mas com grau de pertinência maior na primeira característica.

$4^{\mathbf{a}}$ Comparação As respostas do Investidor 4 para o API do Banco Paulista são:

1-c, 2-c, 3- b, 4- c, 5- b, 6- b, 7- c e 8- b,

com essas respostas foi obtida uma pontuação igual a 20 , logo foi classificado como um investidor moderado. Em seguida, foi pedido para o mesmo responder o API desenvolvido neste trabalho, e as repostas fornecidas ao sistema foram: $36,65,85,100,18$. A classificação do investidor foi de 72.9704 e por esse motivo o mesmo foi classificado como um investidor arrojado. Investimentos associados ao perfil arrojado foram sugeridos a esse investidor.

$5^{\mathbf{a}}$ Comparação As respostas do Investidor 5 para o API do Banco Paulista são:

$$
\text { 1- b, 2- d, 3- c, 4- b, 5- a, 6- c, 7- c e 8- a, }
$$

com essas respostas foi obtida uma pontuação igual a 19, logo foi classificado como um investidor moderado. Em seguida, foi pedido para o mesmo responder o API desenvolvido neste trabalho, ou seja, as questões indicadas na Figura 4 e, neste caso, as respostas fornecidas foram: 12, 50, 75, 40, 06. A classificação do investidor foi de 32.731 e por esse motivo o mesmo foi classificado como 
um investidor moderado. Portanto, os investimentos do perfil moderado foram oferecidos a esse investidor.

$6^{\mathbf{a}}$ Comparação As respostas do Investidor 6 para o API do Banco Paulista são:

$$
\text { 1- d, 2- b, 3- b, 4- c, 5- a, 6- b, 7- a e 8- b, }
$$

com essas respostas foi obtida uma pontuação igual a 14, logo foi classificado como um investidor moderado. Em seguida, foi pedido para o mesmo responder o API desenvolvido neste trabalho, ou seja, as questões indicadas na Figura 4 e, neste caso, as respostas fornecidas foram: 36, 40, 30, 60, 38. A classificação do investidor foi de 32.908 e por esse motivo o mesmo foi classificado como um investidor moderado. Novamente, investimentos do perfil moderado serão sugeridos a esse investidor.

$7^{\mathbf{a}}$ Comparação As respostas do Investidor 7 para o API do Banco Paulista são:

$$
\text { 1-c, 2- a, 3- a, 4- a, 5- a, 6- a, 7- a e 8- b, }
$$

com essas respostas foi obtida uma pontuação igual a 5 , logo foi classificado como um investidor conservador. Em seguida, foi pedido para o mesmo responder o API desenvolvido neste trabalho, ou seja, as questões indicadas na Figura 4 e, neste caso, as respostas fornecidas foram: $24,0,30,10,24$. A classificação do investidor foi de 11.2481 e por esse motivo ele foi classificado como um investidor conservador e moderado, no entanto nota-se que há uma pertinência consideravelmente maior com relação ao perfil conservador. Portanto, o sistema indicou investimentos adequados a ambos perfis.

$8^{\mathbf{a}}$ Comparação As respostas do Investidor 8 para o API do Banco Paulista são:

$$
\text { 1- c, 2- b, 3- b, 4- b, 5- b, 6- a, 7- a e 8- c, }
$$

com essas respostas foi obtida uma pontuação igual a 13, logo foi classificado como um investidor moderado. Em seguida, foi pedido para o mesmo responder o API desenvolvido neste trabalho, ou seja, as questões indicadas na Figura 4 e, neste caso, as respostas fornecidas foram: $24,05,02,06,24$. A classificação do investidor foi de 11.2481 e por esse motivo o mesmo foi classificado como um investidor conservador e moderado, no entanto nota-se que há uma pertinência consideravelmente maior com relação ao perfil conservador. Portanto, novamente, o sistema indicou investimentos adequados a ambos perfis.

\section{Conclusão}

Este trabalho apresentou um modelo teórico baseado em regras fuzzy, o qual classifica o perfil de um investidor. A construção do modelo proposto se fundamentou na experiência de um especialista na área de finanças e considerou a subjetividade da classificação. O uso do sistema baseado em regras fuzzy permite a classificação do perfil de um investidor em mais de uma classe, já em APIs tradicionais, por exemplo o API do Banco Paulista, a diferença de apenas 1 ponto o classifica em uma classe específica e tal situação pode favorecer o desinteresse em investimentos que podem ser interessantes ao perfil do investidor.

O modelo teórico aqui proposto permite uma classificação mais ampla, favorecendo assim a oferta de potenciais investimentos para o investidor, situação a qual, segundo o especialista na área de finanças, é mais interessante tanto para o investidor quanto para bancos e corretoras de investimento.

\section{Agradecimentos}

Agradecemos a UTFPR pelo suporte no desenvolvimento do trabalho.

\section{Referências}

Banco Paulista (2016). Questionário API. Banco Paulista, URL https://www.socopa.com.br/wp-content/ uploads/2018/01/QuestionarioAPI.pdf.

Barros, L. C., Bassanezi, R. C. (2006). Tópicos de lógica fuzzy e biomatemática. Grupo de Biomatemática, Instituto de Matemática, Estatística e Computação Científica (IMECC), Campinas.

Bellucci, D. P., Brandão, A. J. V. (2010). Análise de características empreendedoras utilizando sistemas baseados em regras fuzzy. Tendências em Matemática Aplicada, 11, 133-140.

Cichini, F. A. L. (2009). Aplicação de um sistema de inferência fuzzy de suporte à decisão para estimação de valores de ações cotadas na bolsa de valores de São Paulo. Dissertação de Mestrado, UNESP, Universidade Estadual Paulista, Bauru, SP.

MathWorks Inc. (2016). Matlab 2016. MathWorks Inc., URL https://www.mathworks.com/products/ matlab. html.

Portal do Investidor (2016). Tipos de investimento. URL http://www.portaldoinvestidor.gov.br.

Santos, L. K. C. F. R., Prata, R. A. C. (2016). Análise da malária no estado do amazonas através de sistema de base de regras fuzzy. Em: Livro de resumos do IV Congresso Brasileiro de Sistemas Fuzzy, SBMAC, vol 1, pp. 1-11. 
Sena, F. C. B. (2015). Controle fuzzy aplicado à otimização de um sistema eólico de velocidade variável. Dissertação de Mestrado, UFERSA, Universidade Federal Rural do Semi-Árido, Mossoró, RN.

\section{Contribuição dos autores}

Tais Mara dos Santos

Contribuiç̃̃es substanciais para a concepção do trabalho; análise e

interpretação de dados; criação de novo software usado no trabalho;

redigiu o trabalho e realizou uma revisão substancial.

Michele Cristina Valentino

Contribuições substanciais para a concepção do trabalho; análise e

interpretação de dados; criação de novo software usado no trabalho;

redigiu o trabalho e realizou uma revisão substancial.

Douglas Azevedo

Análise e interpretação de dados; redigiu o trabalho e realizou uma

revisão substancial.

Danilo Peixoto Bellucci

Análise e interpretação de dados. 